\title{
Design and Experimental Investigation of Airfoil for Extruded Blades
}

\author{
Palanisamy Mohan Kumar ${ }^{1}$, Mohan Ram Surya ${ }^{1}$, Ng Pei $\operatorname{Sin}^{1}$, Narasimalu Srikanth ${ }^{1}$
}

\begin{abstract}
Small scale vertical axis wind turbines are always a subject of interest especially for urban installations. Cost and safety are the vital parameters that drive the design of these turbines. Manufacturing of conventional lift driven blades of Darrieus wind turbines incur high cost due to resin transfer moulding or pultrusion process. A novel low cost manufacturing process has been proposed by reinforcing aluminum extruded blades with standard shapes of composite material. Hence this study presents the design of new airfoil profile with desirable characteristics to accommodate glass fiber or carbon fiber tubes without compromising the airfoil qualities such as self-starting capability, high lift to drag ratio at low Reynolds number and sharp trailing edge. The airfoil profile was generated by prescribing the target pressure distribution obtained from the seed airfoil (NACA 0018) optimized by employing Genetic Algorithm (GA) by imposing constraints on minimum thickness, thickness distribution, lift and drag coefficient. The designed airfoil was validated experimentally through wind tunnel tests at Reynolds number $8 \times 10^{4}, 2 \times 10^{5}$ and $3 \times 10^{5}$.
\end{abstract}

Keywords - Airfoil, vertical axis, genetic algorithm, blade, extrusion.

\section{INTRODUCTION}

Unprecedented growth in global population accompanied by appetite for energy, which is expected to be doubled by 2050, exerts demands on energy resources. Depletion of fossil fuels, rise in greenhouse gases concentration and the associated global warming seeks alternative and clean energy sources. Wind energy is one of the promising, affordable and abundant alternative energy with recent annual growth rate of $34 \%$ [1]. Locations around the world with optimum wind speed of more than $7 \mathrm{~m} / \mathrm{s}$ are exploited to the fullest by large sized wind turbines of MW scale thus driving the wind energy sector to shift their focus of attention towards tapping energy from low wind speed of less than $7 \mathrm{~m} / \mathrm{s}$ prevalent in tropical countries [1]. Unfortunately, most of the developing nations are located in tropical regions with low wind speed regime and Small Wind Turbines (SWT) will be a viable solution to plug the energy gap if the cost of energy $(\$ / \mathrm{kW} \mathrm{h})$ is reduced. SWT has swept area less than $200 \mathrm{~m}^{2}$ and power typically around $1-50 \mathrm{~kW}$ and are extensively suited for distributed and micro power generation and can be a potential and competitive

${ }^{1}$ Energy Research Institute, Nanyang Technological University, Singapore, Email: pmkumar@ntu.edu.sg substitute for stand-alone diesel generation for off grid regions. Vertical axis wind turbines are preferred over horizontal axis wind turbines for built environment due to their advantages such as

- Simple design

- Generator and the electrical components are placed on the ground for easy maintainability

- Low noise due to low tip speed ratio

- Blades can be manufacture at a less cost compared to twisted blade of horizontal axis wind turbines

The vertical axis turbines will be even more lucrative if there is a possible way to make inexpensive blades with low cost material. Aluminum blades can be manufactured through extrusion process, yet they are poor in fatigue performance which leads to premature failure of turbine blades. The proposed manufacturing method will increase the fatigue performance of aluminum blades by integrating composite material as a central spar web which will be the primary load carrying member. To favor the integration, the airfoil should have minimum thickness of $18 \% \mathrm{c}$ and the thickness distribution should be at least $40 \%$ of the chord length as per the previous studies on enhancing the fatigue strength of blades. The conventional airfoils such as NACA 4 series family have required thickness, but thickness distribution is not sufficient to incorporate a rectangular spar web. Hence the study is dedicated to design an airfoil for use in vertical axis small wind turbines in the Reynold's number (Re) range of $8 \times 10^{4}$ to $4 \times 10^{5}$.

\section{Design Of NTU-20-V-AIRFOIL.}

\section{A. Desirable Aerodynamic Features}

Albeit the objective is to design an airfoil with enhanced structural properties, the aerodynamic characteristics are vital. Following are the airfoil geometry parameters that has major influence on the aerodynamic properties

Camber : Though the widely used airfoils for vertical axis wind turbines are symmetrical ,cambered airfoils do have their presence especially in smaller turbines .The cambered airfoil aid in self-starting the turbine due to wider drag bucket and thus it address a the major drawback of Darrieus type turbines especially in low Re numbers in the range of $8 \times 10^{4} \sim 2 \times 10^{5}$. The cambered airfoil produces high lift in low $\mathrm{Re}$ and the performance reduces significantly in higher Re above $3 \times 10^{5}$ [2] .Hence the goal of the current study is to design a symmetrical airfoil .

Leading Edge Radius: An airfoil with larger leading edge 
are less sensitive to the roughness especially for smaller vertical axis turbines with low Re number.

Trailing Edge: A sharp trailing edge has greater influence on the drag coefficient of an airfoil. Desirable airfoil geometry should have a larger leading edge and a sharper leading edge [2].

Thickness: The previous study indicates that the generated torque is proportional to the thickness of the airfoil [3]. The airfoil with $19 \%$ chord(c) thickness generates maximum start up torque. Hence the desirable thickness can be around $18 \sim 20 \%$ c. The seed airfoil selected should have these characteristics close to the target airfoil. Thicker airfoil produces less noise compared to the thin airfoils at same tip speed [3]..

\section{B. Design Methodology}

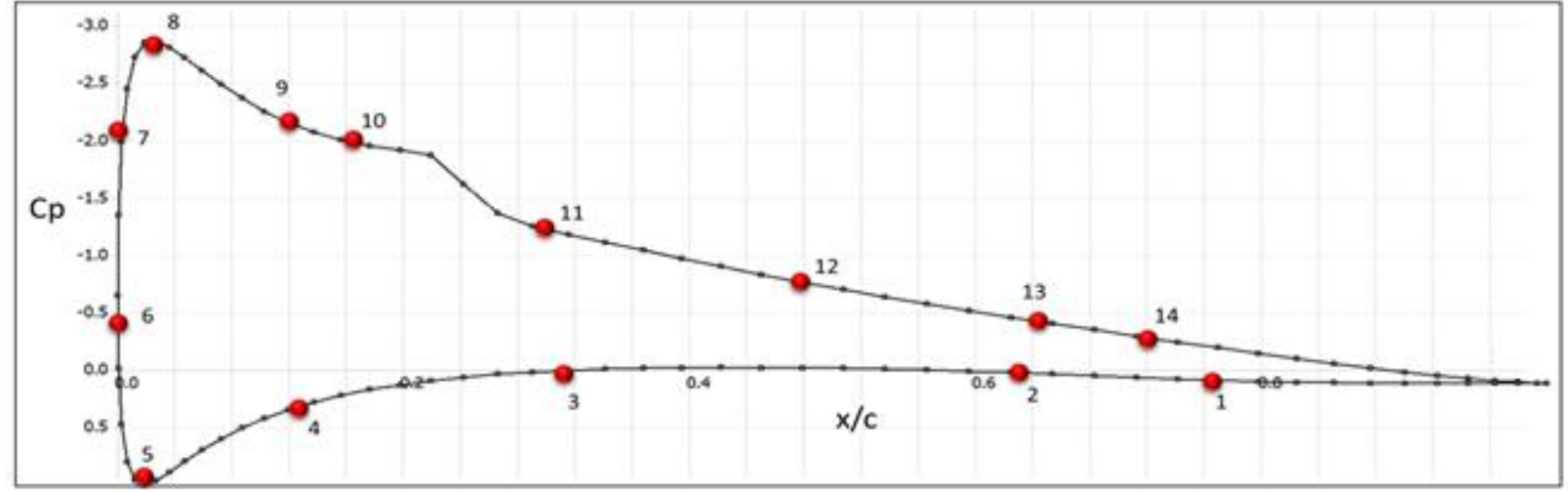

Fig. 1: Pressure distribution of NACA0018 with control

The Re range $\left(6 \times 10^{4}-3 \times 10^{5}\right)$ is based on the typical operating parameters of a 3 straight bladed Small Wind Turbine (SWT) of rotor diameter $2 \sim 4 \mathrm{~m}$, tip speed ratio of $3 \sim 5 \sim$ operating at a freestream wind velocity of $5-8 \mathrm{~m} / \mathrm{s}$. The flow phenomena at this range of Re is complex and airfoil characteristics are dominated by the behaviour of laminar separation bubble [4]. The complexity involved in of an airfoil at this low Re is further compounded by the large variation of Angle of Attack (AoA) at different azimuthal angle. An initial pressure distribution and the corresponding geometry (seed airfoil) is required for the inverse design procedure to begin with the iterative process. The geometry of the seed airfoil will be modified iteratively based on the initial and target pressure difference. In consideration to these favourable airfoil geometry parameters, NACA 0018 will be a viable candidate as the seed airfoil to start with. Iterative design process is repeated until the generated airfoil profile satisfies all the design requirements. An airfoil with such performance characteristics similar to the design requirements can be a good starting point.

\section{Coding}

Genetic Algorithm (GA) has been successfully applied in myriad aeronautical shape optimization in the past [5]. A robust algorithm and less computational efforts are the key factors to optimize the target pressure distribution based on the imposed constraints. Every optimization problem should be represented in GA terms to begin with. An individual is the candidate of interest. Each individual is characterized by set of parameters known as genes and a cluster of genes form a chromosome. Collection of individuals is known as population. The choice of an individual to reproduce further is determined from the fitness value. Individuals are randomly selected from the population and all allowed to reproduce offspring's, which will form the next generation. The process of reproduction happens by crossover and mutation. Crossover selects two individuals and rearranges the chromosome of another individual randomly at crossing site. Crossover avoids the duplication of parents passing to next generation as such. Mutation is performed on the child after crossover, where the child's parameters are altered randomly within the prescribed range [6].

For the current study, pressure distribution is the individual and can be represented by control points as parameters as shown in the Fig. 1. The control points are obtained through the B-spline parametrization method by merging the first and last point to depict a polygon. The pressure distribution curve is divided into upper and lower curves corresponding to the pressure distribution on the pressure side and the suction side The upper curve is represented by 8 points and the pressure side curve is parameterized by 6 points and are free to move within the prescribed bound of values. The co-ordinates of the control points are the design variables for GA and thus 14 variables needs to be optimized.The initial population will be randomly generated with different $\mathbf{x}$ and $\mathbf{y}$ coordinates for the bound values of $\mathbf{x}$ from 0 to 1 and $\mathbf{y}$ from -3 to 1 .

The optimized pressure distribution will be generated by GA and passed to Xfoil. Xfoil is capable of generating airfoil geometry profile through the full inverse design module from the obtained pressure distribution and compute lift coefficient $\left(\mathrm{C}_{\mathrm{l}}\right)$, drag coefficient $\left(\mathrm{C}_{\mathrm{d}}\right)$ and the corresponding thickness.GA with the capability of multi objective optimization of NSGA-2 embedded in the MATLAB math works was used to run the optimization. The whole process of GA is illustrated in Fig. 2. 


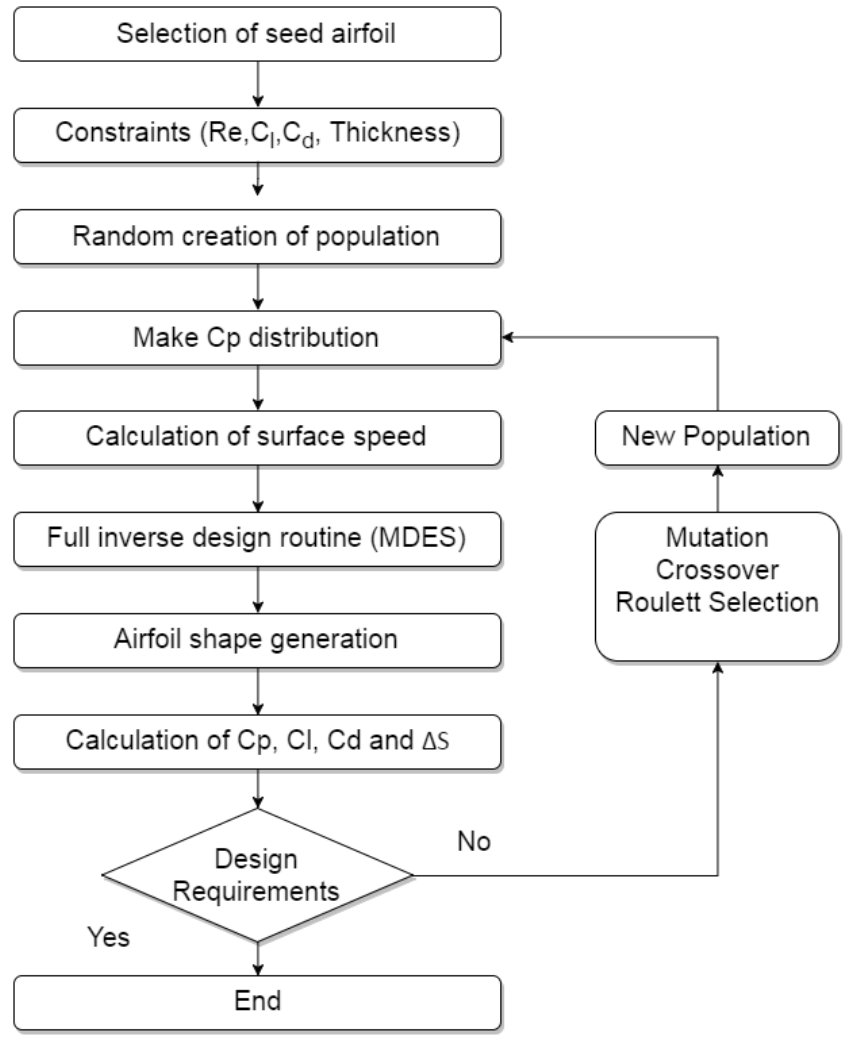

Fig.2: Genetic Algorithm Flow Diagram.

\section{Objective Function and Constraints}

The goal of GA is to obtain maximum fitness value. The fitness function is derived from the design variables combined with proper weightage. The weightage factor k may vary from 0 to 1 . Every individual is evaluated for the fitness value to be qualified for reproduction. The GA optimization process will evaluate the fitness function and to obtain a maximum value based on the following constraints.

- Reynolds number

- Thickness of the airfoil

- Thickness distribution along the chord length

- Modification to the seed airfoil geometry

Deciding a proper weightage for the above constraints may be complicated as the weightage proposition will have a greater influence on outcome of fitness function value. For airfoil, aerodynamic and structural properties are the key

parameters that have to be enhanced. A structurally sound airfoil will have a thick geometry which has poor aerodynamic characteristics. Thus an optimum thickness exists for every application of expected aerodynamic properties. As the angle of attack typically varies from $0^{\circ}$ to $\pm 180^{\circ}$, the generated lift and drag forces induces alternative stresses that affects the fatigue strength of blades [7]. Hence the fitness function should have both aerodynamic component and structural component. Sections of airfoil geometry can be retained by the confined movement of control points. In the current study the leading edge of the seed airfoil should be retained with minimum movements as a well-defined leading edge will delay the deep stall at larger angle of attack. The delay in deep stall also limits the drop of lift coefficient, though the Re number and turbulent intensity are also contributing factors. The difference in the control point co-ordinates at the leading edge portion of seed airfoil pressure distribution and the newly generated pressure distribution are summed and added to the fitness function with negative weightage to preserve leading edge geometry as shown in equation (1).

$$
\Delta s=\sum_{m=6}^{10}\left|x_{f}(m)-x_{g}(m)\right|+\sum_{m=6}^{10}\left|y_{f}(m)-y_{g}(m)\right|
$$

In the Eq.1 , $\mathrm{x}_{-}(\mathrm{f}$,$) and \mathrm{y}_{-}(\mathrm{f})$ are the control points $\Delta \mathrm{s}$ is the difference between newly generated control points with previous points .For the NACA series airfoils the thickness reaches maximum at certain position of the chord length and gradually decreases up to trailing edge as shown in Fig. 3. For an extruded blade the thickness has to be extended over $40 \%$ of the chord length. Increase in thickness distribution for a longer chord length will have more viscous drag which tends to decrease the $\mathrm{Cl} / \mathrm{Cd}$.

Hence a thicker airfoil will have more drag at lower AoA resulting in lower $C_{l} / C_{d}$, whereas a thinner airfoil will have higher aerodynamic efficiency due to decrease in adverse pressure gradient on the upper surface which hamper the flow separation, but structurally weak. When the $\mathrm{k}$ value was set close at 0 , the $C_{l} / C_{d}$ value will be maximum for the input $R e$ number, but the thickness will be distribution length will be shorter. On the other hand, the $\mathrm{k}$ value close to 1 will generate an airfoil with lower $C_{l} / C_{d}$ value but with longer thickness distribution length. Trailing edge thickness and the transition point locations are free variables. The objective function is defined in the equation (2). $t_{c}$ and $t$ are thickness distribution and maximum thickness.

$$
\mathrm{F}=k / 3\left(\frac{C_{l}}{C_{D}}\right)+k / 3(\mathrm{t})+k / 3\left(t_{c}\right)+(1-k) \Delta s
$$

Reynold's number. An inbuilt smoothing technique in MDES was applied on the velocity distribution generated from pressure distribution optimized by GA before creating the airfoil geometry, to avoid a distortion or glitch in the surface by modifying the Hanning filter.

\section{EXPERIMENTAL SETUP}

The aerodynamic characteristics of the designed airfoil are validated by wind tunnel tests performed at NTU-low turbulence subsonic wind tunnel facility as shown in fig. 5 . The open return wind tunnel has rectangular test section size of $960 \mathrm{~mm}$ x $960 \mathrm{~mm}$ and maximum velocity up to $30 \mathrm{~m} / \mathrm{s}$. A low turbulence level of $0.2 \%$ in the test section was achieved 


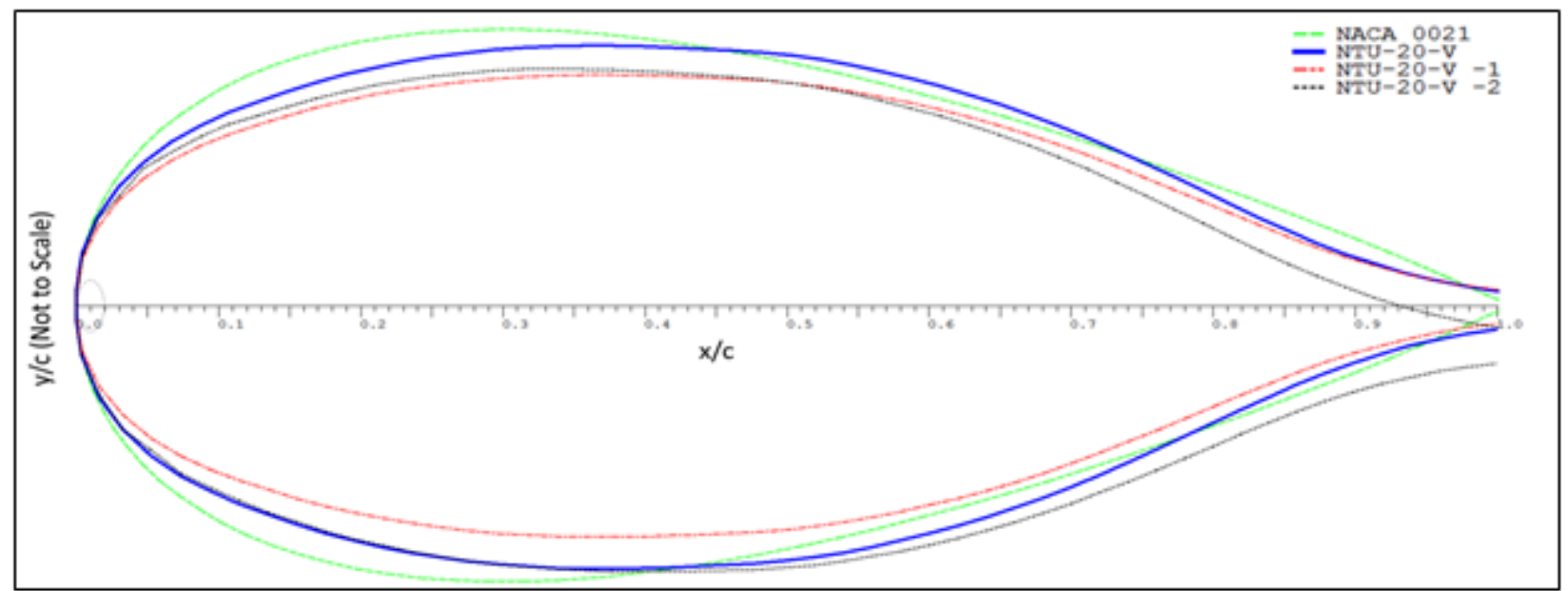

Fig. 3: Contours of various airfoils during optimization process

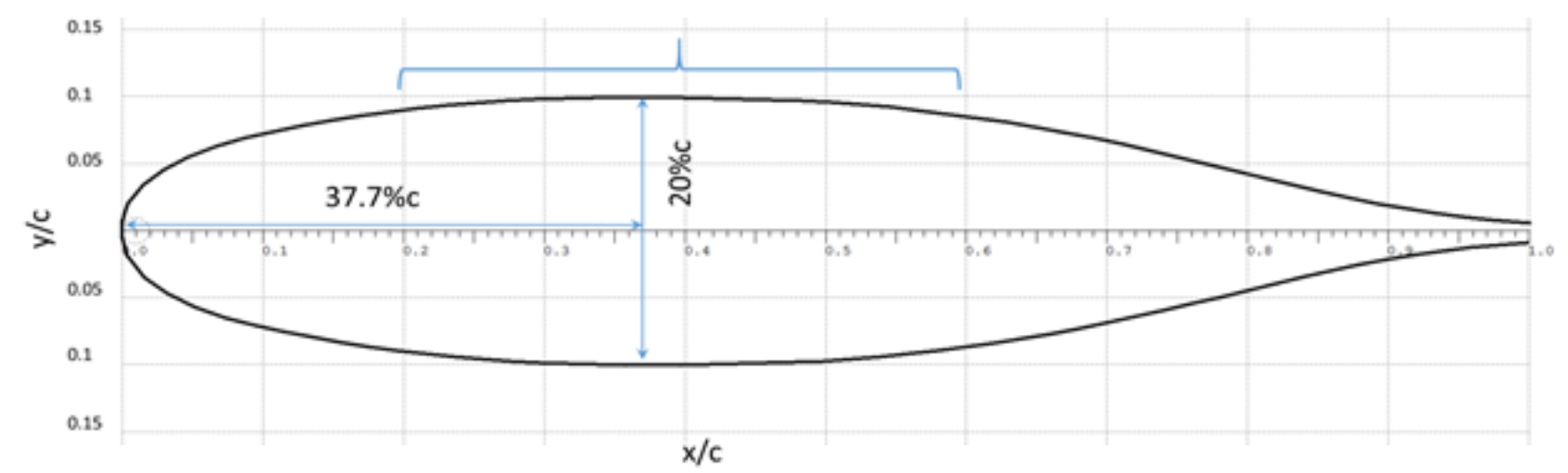

Fig.4: Airfoil profile smoothing
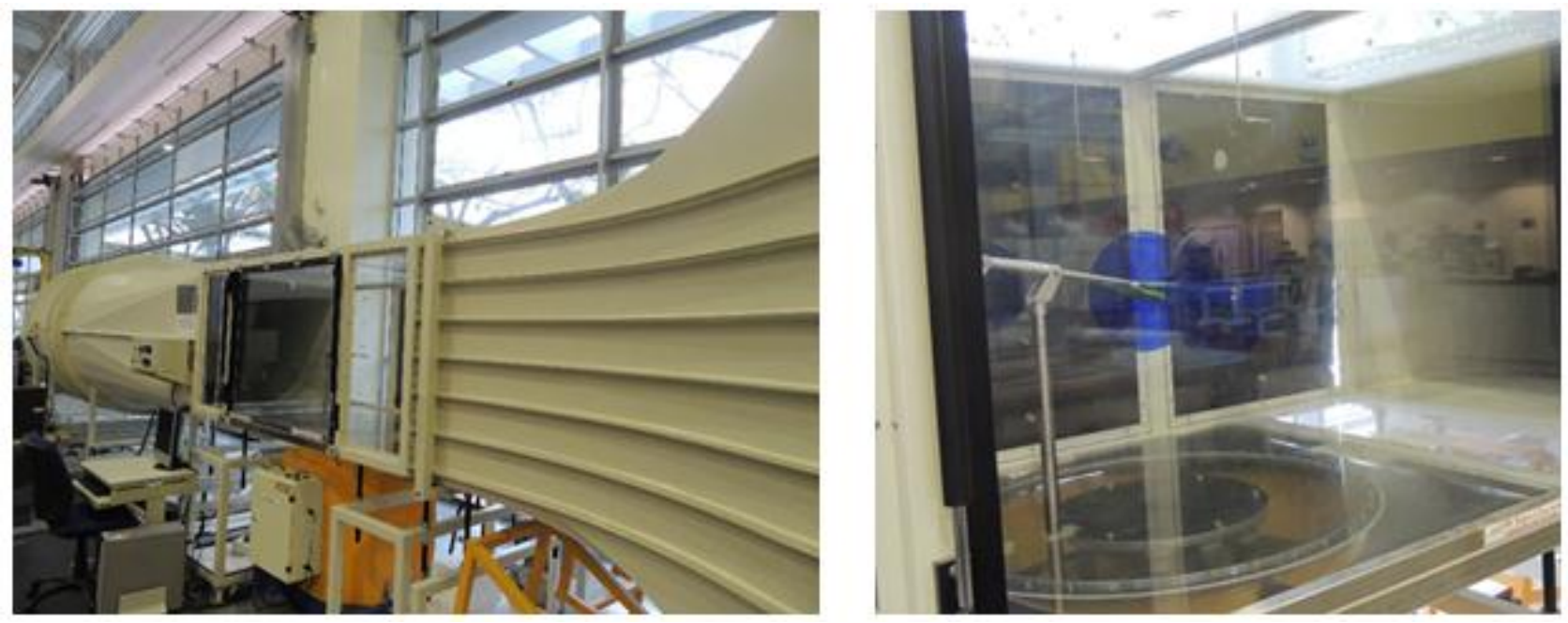

Fig.5: Wind tunnel testing of NTU-20-V airfoil in the wind tunnel. 

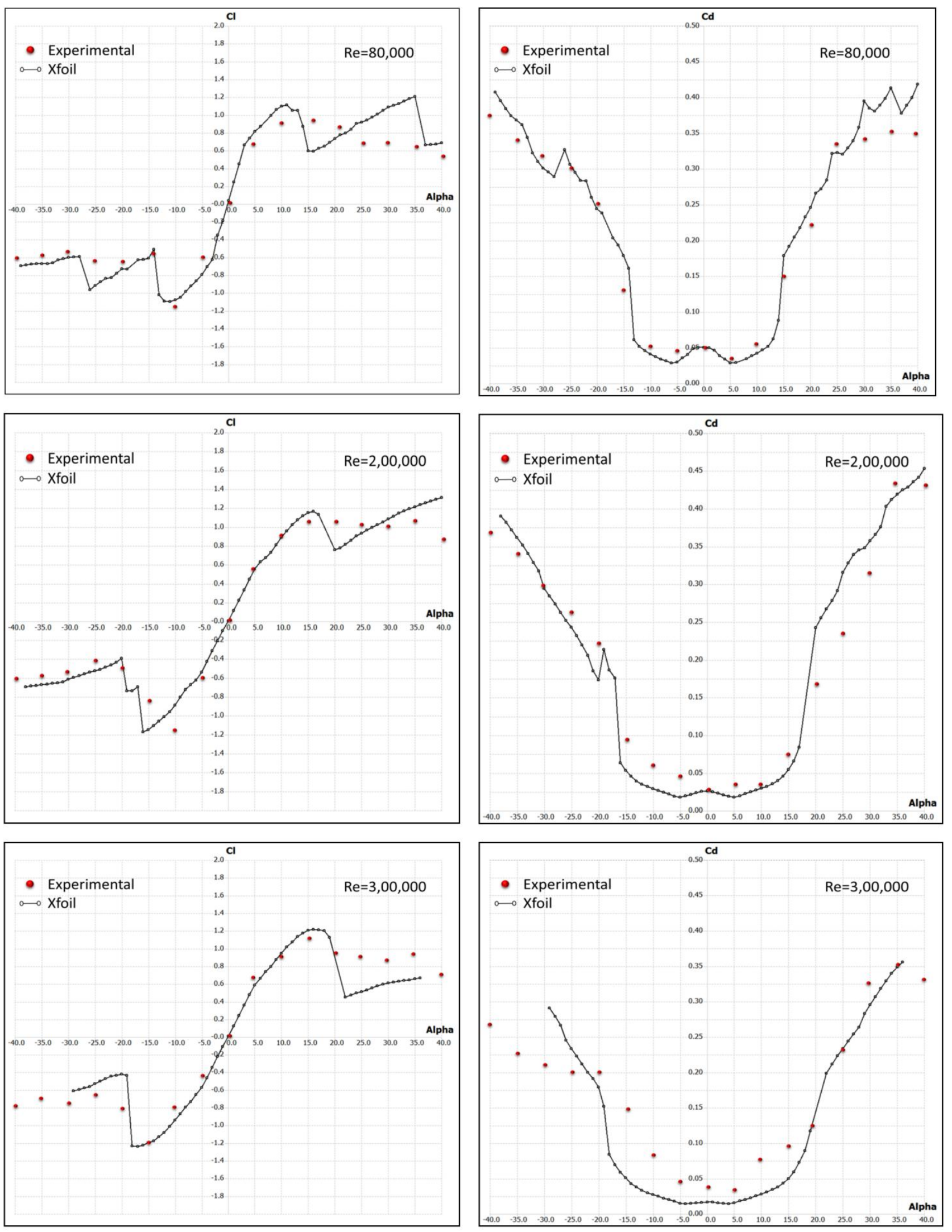

Fig. 6: $C_{1}$ and $C_{d}$ of NTU-20-V airfoil at different 
by incorporating a fine honeycomb flow straighter in the suction chamber. The airfoils for the wind tunnel test are 3D printed with the chord length of $200 \mathrm{~mm}$ and span of $250 \mathrm{~mm}$.

Surface roughness of printed models are improved by repeated polishing and painting to avoid the flow separation incited by the roughness especially in the lower Re range. The airfoils together with the end plates are mounted on a precision 3-axis force balance sensor, which can automatically change the angle of attack ( $-45^{\circ}$ to $45^{\circ}$ ) as per the input data. End plates of diameter $250 \mathrm{~mm}$ were mounted on either side of the airfoils to ensure the span wise flow uniformity. The output from the force balance and the pitot tube are logged by LabVIEW software. The data logged are free of blockage corrections as the airfoil volume is less than $20 \%$ of the wind tunnel cross section. These are uncertainties and errors in the scope of experiment and may deviate from the numerical validation results.

\section{RESUlTS AND DiscUSSION}

The aerodynamic characteristics of the designed airfoil are obtained for the Re numbers of $8 \times 10^{4}, 2 \times 10^{5}$ and $3 \times 10^{5}$. During testing, some low amplitude vibrations starts to creep in at higher AoA of more than $\pm 30^{\circ}$. Hence the lift and drag coefficient obtained in that range of AoA differs much compared to Xfoil predictions. The drag forces for all three Re numbers by the end plate alone was measured and corrections are made in the total drag measured by the force balance sensor.

For $\operatorname{Re} 8 \times 10^{4}$, the Xfoil predictions are close to the experimental value from $-10^{\circ}$ to $5^{\circ}$.For other AoA the value deviated from Xfoil as it is not able to predict in such low Re due to complex fluid behaviour and associated flow separation .The lift and drag coefficients of NTU-20-V at $8 \times 10^{4}$ is plotted in the Fig.6. Maximum lift coefficient of 1.1 was obtained at AoA of $11^{\circ}$. The lift and drag coefficients for the Re $2 \times 10^{5}$ are plotted in the Fig 7. Xfoil predicts higher lift coefficient close to 1.2 and then a sudden drop, whereas the experimental results exhibit a gradual drop in lift. The gradual loss of lift tends to be a smoother stall at AoA of $15^{\circ}$.For the $\operatorname{Re} 3 \times 10^{5}$, maximum lift coefficient of 1.2 was predicted by Xfoil, whereas the experimental value is at 1.1. The drag coefficients for three Re numbers are low for the AoA from $-15^{\circ}$ to $15^{\circ}$ and drastically increase after that.

\section{V.CONCLUSION}

A new airfoil was designed to aid in manufacturing of vertical axis wind turbine blades through extrusion process. An attempt has been made to enhance the structural characteristic of commonly employed NACA 4 series airfoils. The designed airfoil NTU-20-V was subjected to wind tunnel test for the Re numbers $8 \times 10^{4}, 2 \times 10^{5}$ and $3 \times 10^{5}$ and compared with Xfoil predictions as shown in Fig. 6,7 and 8. The results show that a maximum lift coefficient of 1.1 was obtained at AoA of $15^{\circ}$ for $\operatorname{Re} 8 \times 10^{4}$ in Fig. $6,1.17$ at Re $2 \times 10^{5}$ in Fig. 6 and 1.2 at $\operatorname{Re} 3 \times 10^{5}$ in Fig. 6. The experimental values closely follow the Xfoil predications in smaller AoA and shows a smooth stall at higher AoA in which Xfoil contradicts. Albeit the aerodynamic characteristics of the designed airfoil were inferior to NACA 0018, the structural properties are expected to be superior.

\section{FUTURE WORK}

The future work includes the wind tunnel testing of small vertical axis wind turbine incorporating the designed NTU-20$\mathrm{V}$ airfoil and NACA 0018 to determine the power coefficient and to compare the aerodynamic performance under similar conditions. Furthermore, a detailed investigation will be carried out to evaluate the improvement in structural characteristics such as maximum bending moment, blade natural frequency and fatigue.

\section{ACKNOWLEDGMENT}

This research is supported by the National Research Foundation, Prime Minister's Office, Singapore under its Energy Innovation Research Programme (EIRP Award No. NRF2013EWT-EIRP003-032: Efficient Low Flow Wind Turbine).

\section{REFERENCES}

[1] B.R. Karthikeya, Prabal S. Negi, N. Srikanth, "Wind resource assessment for urban renewable energy application in Singapore", Renewable Energy, Volume 87, Part 1, March 2016, Pages 403-414.

[2] P. G. Migliore, W. P. Wolfe, and J. B. Fanucci. "Flow Curvature Effects on Darrieus Turbine Blade Aerodynamics", Journal of Energy, Vol. 4, No. 2 (1980), pp. 49-55.

[3] Todd Mosher. "Conceptual Spacecraft Design Using a Genetic Algorithm Trade Selection Process", Journal of Aircraft, Vol. 36, No. 1 (1999), pp. 200-208.

[4] Ben Gardner and Michael Selig. "Airfoil Design Using a Genetic Algorithm and an Inverse Method", 41st Aerospace Sciences Meeting and Exhibit, Aerospace Sciences Meetings.

[5] Lin Wang, Xiongwei Liu, Athanasios Kolios, "State of the art in the aeroelasticity of wind turbine blades: Aeroelastic modelling", Renewable and Sustainable Energy Reviews, Volume 64, October 2016, Pages 195-210.

[6] Li, Q., Maeda, T., Kamada, Y. et al. "Analysis of aerodynamic load on straight-bladed vertical axis wind turbine". Journal of. Therm. Sci. (2014) pp.23: 315.

[7] Van Rooij R, Timmer WA. "Roughness sensitivity considerations for thick rotor blade airfoils". Journal of Solar Energy Engineering 2003; 125: pp.468-478. 